\title{
Analysis of clinically relevant somatic mutations in high-risk head and neck cutaneous squamous cell carcinoma
}

Catherine Zilberg ${ }^{1}$, Matthew Weicai Lee ${ }^{1}$, Bing Yu ${ }^{1,2}$, Bruce Ashford ${ }^{3,4,5}$, Spiridoula Kraitsek ${ }^{2}$, Marie Ranson ${ }^{4,5,6}$, Kerwin Shannon ${ }^{7}$, Mark Cowley ${ }^{8,10}$, N Gopalakrishna Iyer ${ }^{9}$, Carsten E Palme ${ }^{1,7}$, Sydney Ch'ng ${ }^{1,7}$, Tsu-Hui(Hubert) Low ${ }^{1,7}$, Sandra O'Toole ${ }^{1,11}$, Jonathan R Clark ${ }^{1,7}$ and Ruta Gupta ${ }^{1,11}$

${ }^{1}$ Central Clinical School, The University of Sydney, Sydney, Australia; ${ }^{2}$ Department of Medical Genomics, Royal Prince Alfred Hospital, Sydney, Australia; ${ }^{3}$ Illawarra and Shoalhaven Local Health District (ISLHD), Wollongong, Australia; ${ }^{4}$ School of Biological Sciences, University of Wollongong, Wollongong, Australia; ${ }^{5}$ Illawarra Health and Medical Research Institute (IHMRI), Wollongong, Australia; ${ }^{6}$ Centre for Oncology Education and Research Translation (CONCERT), Liverpool, Australia; ${ }^{7}$ The Sydney Head and Neck Cancer Institute, Chris O'Brien Lifehouse, Sydney, Australia; ${ }^{8}$ Kinghorn Cancer Centre and Garvan Institute of Medical Research, Sydney, Australia; ${ }^{9}$ Singhealth/Duke-NUS Head and Neck Center, National Cancer Center Singapore (NCCS), Singapore; ${ }^{10}$ St Vincent's Clinical School, UNSW Sydney, Sydney, Australia and ${ }^{11}$ Tissue Pathology and Diagnostic Oncology, Royal Prince Alfred Hospital, Sydney, Australia

\begin{abstract}
Cutaneous squamous cell carcinoma is the second most prevalent malignancy, most frequently occurring in the head and neck (head and neck cutaneous squamous cell carcinoma). Treatment of locally advanced or metastatic disease is associated with functional morbidity and disfigurement. Underlying genetic mechanisms are poorly understood. Targeted sequencing of $\mathbf{4 8}$ clinically relevant genes was performed on DNA extracted from formalinfixed and paraffin-embedded high-risk primary head and neck cutaneous squamous cell carcinomas that remained non-metastatic at minimum follow-up of 24 months. Associations of somatic mutations with clinicopathologic characteristics were evaluated and compared with those described in the literature for metastatic disease. Alterations in 44 cancer-associated genes were identified. TP53 was mutated in $100 \%$ of cases; APC, ATM, ERBB4, GNAQ, KIT, RB1 and ABL1 were altered in $60 \%$ of cases. FGFR2 mutations (40\%) were exclusively seen in patients with perineural invasion. MLH1 mutations were exclusively seen in the two younger patients ( $<45$ years). Lower incidences of $\mathrm{NOTCH} 1$ mutations were observed compared with that described in metastatic head and neck cutaneous squamous cell carcinoma in the literature. Somatic mutations susceptible to EGFR inhibitors, and other small molecular targeted therapeutics were seen in $60 \%$ of cases. This study provides insights into somatic mutations in non-metastatic, high-risk head and neck cutaneous squamous cell carcinoma and identifies potential therapeutic targets. Alterations in FGFR2 and NOTCH1 may have roles in local and distant disease progression.
\end{abstract}

Modern Pathology (2018) 31, 275-287; doi:10.1038/modpathol.2017.128; published online 6 October 2017

Cutaneous squamous cell carcinoma is the second most common malignancy in the world and most frequently occurs in the head and neck. ${ }^{1}$ Disease

Correspondence: Associate Professor $\mathrm{R}$ Gupta, MBBS, MD, FRCPA, Department of Anatomic Pathology and Diagnostic Oncology, Royal Prince Alfred Hospital, Building 94, John Hopkins Drive, Camperdown, NSW 2050, Australia.

E-mail: Ruta.Gupta@sswahs.nsw.gov.au

Received 13 June 2017; revised 10 August 2017; accepted 12 August 2017; published online 6 October 2017 incidence is rising in countries with a high fair skin population and solar ultraviolet index, and with the aging population. ${ }^{2}$ The National Comprehensive Cancer Network (https://www.nccn.org/) guidelines indicate that nearly $14 \%$ of head and neck cutaneous squamous cell carcinomas seen in a Dermatology department are 'high-risk' lesions because of presence of perineural invasion and/or invasion of the subcutaneous tissues. ${ }^{3}$

Surgical resection forms the mainstay of treatment and is followed in selected cases by adjuvant 

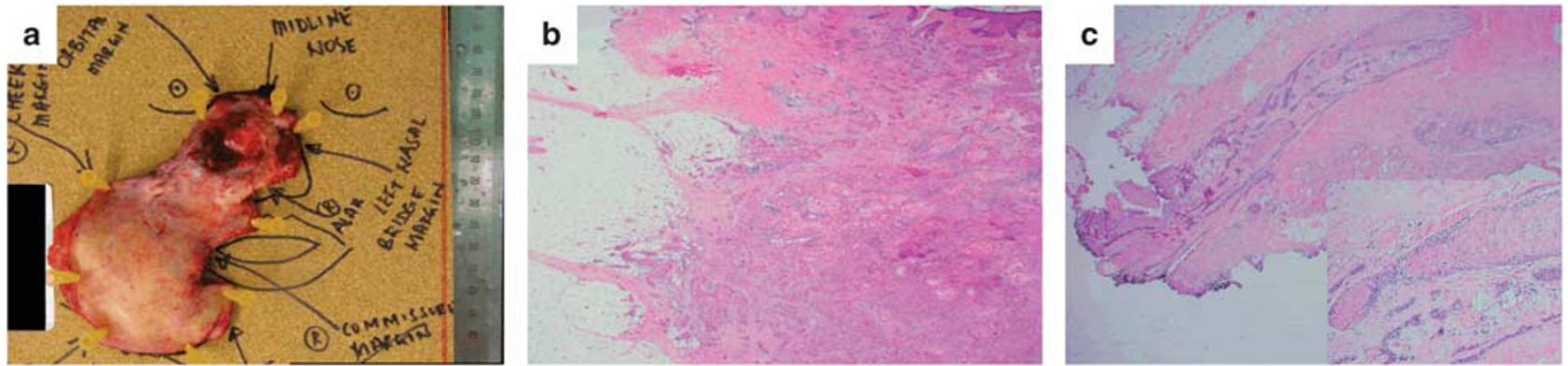

Figure 1 (a) Excision of HNcSCC involving the nasal alae with subcutaneous extension onto the right cheek. The entire tumor including the ulcerated component and the subcutaneous extension measure $55 \mathrm{~mm}$ in maximum dimension. (b) Moderately differentiated squamous cell carcinoma, infiltrating through the dermis and the subcutaneous tissue to a depth of $15 \mathrm{~mm}$, Clark level 5 (hematoxylin and eosin $\times 20$ ). (c) Perineural invasion of a large nerve bundle at the deep margin of the specimen (high risk) (hematoxylin and eosin $\times 40$ ); inset: squamous cell carcinoma is seen within the perineurium, surrounding approximately two-thirds of the circumference of the nerve (hematoxylin and eosin $\times 100$ ).

radiotherapy, unfortunately a significant proportion of head and neck cutaneous squamous cell carcinomas develop local recurrences and approximately $14 \%$ of high-risk lesions develop regional metastases. ${ }^{3}$ Predicting which patients will develop recurrence or metastases is not possible as the currently available clinical and histologic prognostic indicators are unreliable. ${ }^{4}$ Owing to lack of effective second-line therapies, over $30 \%$ of patients with advanced nodal disease and $89 \%$ of patients with distant metastases die from head and neck cutaneous squamous cell carcinoma, and in those cured by radical surgery the quality of life remains poor, highlighting the need for other therapeutic options. ${ }^{5}$

The recent advances in massive parallel sequencing technologies have significantly transformed the treatment and survival in several lethal malignancies such as melanoma and pulmonary adenocarcinoma. ${ }^{6,7}$ The utility of these techniques remains relatively unexplored in head and neck cutaneous squamous cell carcinoma. Herein, we evaluate the somatic mutations in high-risk tumors using a clinically relevant, commercially available, targeted cancer gene panel to ensure universal applicability of our findings. A cohort of patients with high-risk head and neck cutaneous squamous cell carcinoma that did not develop metastases after a minimum follow-up of 24 months was selected for this study to enable comparison with the cohorts of metastatic cutaneous squamous cell carcinoma described in the literature. The primary aims of this study were to identify somatic mutations associated with adverse histopathological features in head and neck cutaneous squamous cell carcinoma and to investigate the differences in somatic mutations observed in this non-metastatic cohort with those described in metastatic head and neck cutaneous squamous cell carcinoma cohorts in the literature. The secondary aim was to identify somatic mutations amenable to currently available clinical and preclinical targeted therapeutic agents.

\section{Materials and methods}

Following institutional human research ethics committee approval, patients with high-risk head and neck cutaneous squamous cell carcinoma treated with curative intent between 2008 and 2014 were identified from the prospectively collected database held at the Sydney Head and Neck Cancer Institute. A representative example of the type of head and neck cutaneous squamous cell carcinoma included in this study is depicted in Figure 1. High-risk disease was defined as per the criteria provided in the 7 th edition of American Joint Commission on Cancer Staging Manual. ${ }^{8}$ Only patients who had either undergone concurrent sentinel node biopsy or neck dissection with at least 24 months of follow-up and complete clinical data were included. Patients with histopathologically positive sentinel lymph node biopsies or concurrent neck dissections and those who developed nodal metastases during follow-up were excluded. In total, 24 cases of highrisk head and neck cutaneous squamous cell carcinoma met the selection criteria.

The histopathology slides and paraffin blocks were retrieved from the archives of the Department of Tissue Pathology and Diagnostic Oncology at Royal Prince Alfred Hospital, Sydney, NSW, Australia. A complete histopathology review was performed and the tumor size, depth of invasion, lymphovascular and perineural invasion, bone involvement and margins of resection were recorded. Highly cellular areas of the tumor with a neoplastic cell content of $30-90 \%$ and without necrosis, keratin, inflammatory infiltrate or hemorrhage were identified.

Malignant tissue selected as described above was macro-dissected from the blocks for deoxyribonucleic acid (DNA) extraction. Case-matched normal tissue from the benign neck lymph nodes was used as germline control for mutation filtering. DNA extraction was performed using truXTRAC ${ }^{\circledR}$ formalin-fixed paraffin-embedded DNA microTUBE kit (Covaris, Woburn, MA, USA) as per the manufacturer's instructions. 
Samples with sufficient DNA that passed the quality control checks using the Illumina formalinfixed paraffin-embedded quality control kit were available in 10 cases. Thus, the final cohort used for library preparation included 10 patients. The TruSeq Amplification Cancer Panel ${ }^{\circledR}$ (Illumina, San Diego, CA, USA) was utilized to assess 48 clinically relevant genes: $A B L 1, A L K, A K T 1, A P C, A T M, B R A F$, CDH1, CDKN2A, CSF1R, CTNNB1, EGFR, ERBB2, ERBB4, FBXW7, FGFR1, FGFR2, FGFR3, FLT3,

Table 1 Patient clinicopathological data

\begin{tabular}{|c|c|c|}
\hline Variable & $\mathrm{N}$ & $\%$ \\
\hline \multicolumn{3}{|l|}{ Age, years } \\
\hline$\leq 55$ & 2 & 20 \\
\hline $56-65$ & 2 & 20 \\
\hline$\geq 66$ & 6 & 60 \\
\hline \multicolumn{3}{|l|}{ Sex } \\
\hline Male & 8 & 80 \\
\hline Female & 2 & 20 \\
\hline \multicolumn{3}{|l|}{ Location } \\
\hline Ear & 2 & 20 \\
\hline Forehead/scalp & 4 & 40 \\
\hline Lip & 2 & 20 \\
\hline Nose & 1 & 10 \\
\hline Pre-auricular & 1 & 10 \\
\hline \multicolumn{3}{|l|}{ Differentiation } \\
\hline Well & 1 & 10 \\
\hline Moderate & 7 & 70 \\
\hline Poor & 2 & 20 \\
\hline \multicolumn{3}{|l|}{ Depth of invasion } \\
\hline$<5 \mathrm{~mm}$ & 3 & 30 \\
\hline $5-10 \mathrm{~mm}$ & 2 & 20 \\
\hline$>10 \mathrm{~mm}$ & 5 & 50 \\
\hline \multicolumn{3}{|l|}{ Pattern of invasion } \\
\hline Pushing & 0 & 0 \\
\hline Infiltrative & 10 & 100 \\
\hline \multicolumn{3}{|l|}{ Margins } \\
\hline Clear & 4 & 40 \\
\hline Close & 1 & 10 \\
\hline Involved & 5 & 50 \\
\hline \multicolumn{3}{|l|}{ Lymphovascular invasion } \\
\hline Yes & 2 & 20 \\
\hline \multicolumn{3}{|l|}{ Perineural invasion } \\
\hline Yes & 4 & 40 \\
\hline \multicolumn{3}{|l|}{ Pathological T category } \\
\hline $\mathrm{T} 1$ & 2 & 20 \\
\hline $\mathrm{T} 2$ & 6 & 60 \\
\hline T3 & 0 & 0 \\
\hline $\mathrm{T} 4$ & 1 & 10 \\
\hline \multicolumn{3}{|l|}{ Pathological N category } \\
\hline No & 10 & 100 \\
\hline \multicolumn{3}{|l|}{ Treatment } \\
\hline Surgery alone & 7 & 70 \\
\hline Surgery + radiotherapy & 3 & 30 \\
\hline
\end{tabular}

GNA11, GNAQ, GNAS, HNF1A, HRAS, IDH1, JAK2, JAK3, KDR, KIT, KRAS, MET, MLH1, MPL, NOTCH1, NPM1, NRAS, PDGFRA, PIK3CA, PTEN, PTPN11, RB1, RET, SMAD4, SMARCB1, SMO, SRC, STK11, TP53 and VHL. The reads were aligned to the human reference genome (GRCh37) using Isis SmithWaterman-Gotoh (v2.6). Illumina Somatic Variant Caller (v4.0) and Illumina variant studio (v2.3) were used for variant calling and annotation, respectively. Somatic variants were identified after deducting the normal/germline variants observed in the matched normal samples from those observed in the tumor samples. Variant positions with a coverage of 500x read depth or more, and those variant alleles observed at a $>5 \%$ frequency were included in further analysis. A single patient (case 1) did not have a matched normal, so variants known to be common in ExAC (MAF>0.1\%) were filtered for this individual.

In-silico analysis using PolyPhen $2^{9}$ and SIFT ${ }^{10}$ was performed. The Catalogue of Somatic Mutations in Cancer (COSMIC) database ${ }^{11}$ was used for functional annotation of the identified variants and to understand their potential interactions in the relevant signal transduction pathways. Functional Analysis through Hidden Markov Models (FATHMM) (http://fathmm.biocompute.org.uk/cancer.html) were used to understand the pathogenicity of the identified somatic alterations. ${ }^{12}$

All functionally characterized mutations that have been shown to promote carcinogenesis in other malignant neoplasms, in animal models and in cell cultures, and all truncations and deletions in tumorsuppressor genes were selected for further analysis.

Literature searches using PubMed for English language literature were performed to identify studies providing the details of somatic mutations in metastatic head and neck squamous cell carcinoma and somatic alterations amenable to currently available therapies.

\section{Results}

\section{Clinicopathologic Characteristics of the High-Risk, Non-Metastatic cSCC Cohort}

The final cohort of 10 patients included 8 men and 2 women with a median age of 71 years (range 38-92 years) at the time of surgery. The median tumor diameter was $25 \mathrm{~mm}$ (range 7-160 mm) with a median depth of invasion of $10.5 \mathrm{~mm}$ (range 2$70 \mathrm{~mm})$ and $4(40 \%)$ patients exhibited perineural invasion (Table 1).

\section{Overview of Somatic Mutations}

Targeted sequencing of 48 cancer-associated genes to a mean coverage of $6907 \pm 2044(n=9)$ in the normal samples and $9544 \pm 6486 \quad(n=10)$ in the tumor samples was performed. 
A total of 1313 mutations were identified across the 10 patient samples. These included 488 mutations in the coding region and 12 essential splice site variations, and 813 mutations in the non-coding region. Of the coding region and splice site mutations, 464 somatic mutations were found to be functionally significant across 44 of the targeted genes by using the COSMIC database, FATHMM scores, literature review and including those mutations leading to truncations or deletions of genes. A mean of 46 (range 3-146) significant somatic mutations were observed per patient. Although the unpaired sample had a higher than average number of mutations, it did not demonstrate the greatest mutation burden (53 putative somatic variants vs range of 3-146). C/T and G/A transitions, characteristic of UV-mediated DNA damage ${ }^{13}$ were the dominant substitution comprising $72 \%$ of the mutation spectrum. In all, $90 \%$ of samples contained at least one $\mathrm{C} / \mathrm{T}$ transition.

The most frequently observed non-synonymous somatic mutations were in TP53 $(N=10,100 \%)$, $A T M(N=6,60 \%), A P C(N=6,60 \%), E R B B 4(N=6$, $60 \%)$, GNAQ $(N=6,60 \%), A B L 1(N=6,60 \%), K I T$ $(N=5,50 \%)$, PIK3CA $(N=5,50 \%), A K T 1 \quad(N=5$, $50 \%)$ and $R B 1(N=5,50 \%)$. An overview of mutated genes and types of alterations identified is provided in Figure 2.

Table 2 lists the validated loss-of-function mutations in tumor-suppressor genes. Eleven mutually exclusive, functionally relevant TP53 mutations were identified in 9 cases-9 of which were within the DNA-binding domain of the gene (Figure 3a). TP53 R282W has been described in cutaneous SCC ${ }^{14}$ and basal cell carcinoma, ${ }^{15}$ whereas the others have been described in breast, colorectal and other carcinomas (Table 2).

Functionally significant alterations in the $A P C$ gene were observed in six patients. These occurred within the mutational cluster region of the gene (codons 1030-1700 containing domains integral to $\beta$-catenin regulation). Two truncating mutations at positions R1114* and Q1328* in the APC gene that are well annotated in colorectal carcinoma ${ }^{16,17}$ were also observed (Figure 3b).

There were three nonsense and two deletion mutations in the ATM gene, including two cases with truncations at R2443* previously reported in mantle cell lymphoma ${ }^{18}$ (Figure 3c). Two patients with functionally relevant $A T M$ mutations also showed loss-of-function mutations in the MLH1 gene (Q407*, Q426*, R423*). These are novel MLH1 mutations, resulting in loss of the C-terminal dimerization domain necessary for normal function of the mismatch repair protein. ${ }^{19}$

The three cases with SMAD4 mutations include: two deletions (c.533delC, S178*) and (c.1186delG, p. D396Mfs*19), and two truncating mutations, K122* and Q448*. SMAD4 deletion in head and neck epithelia has been described to result in spontaneous

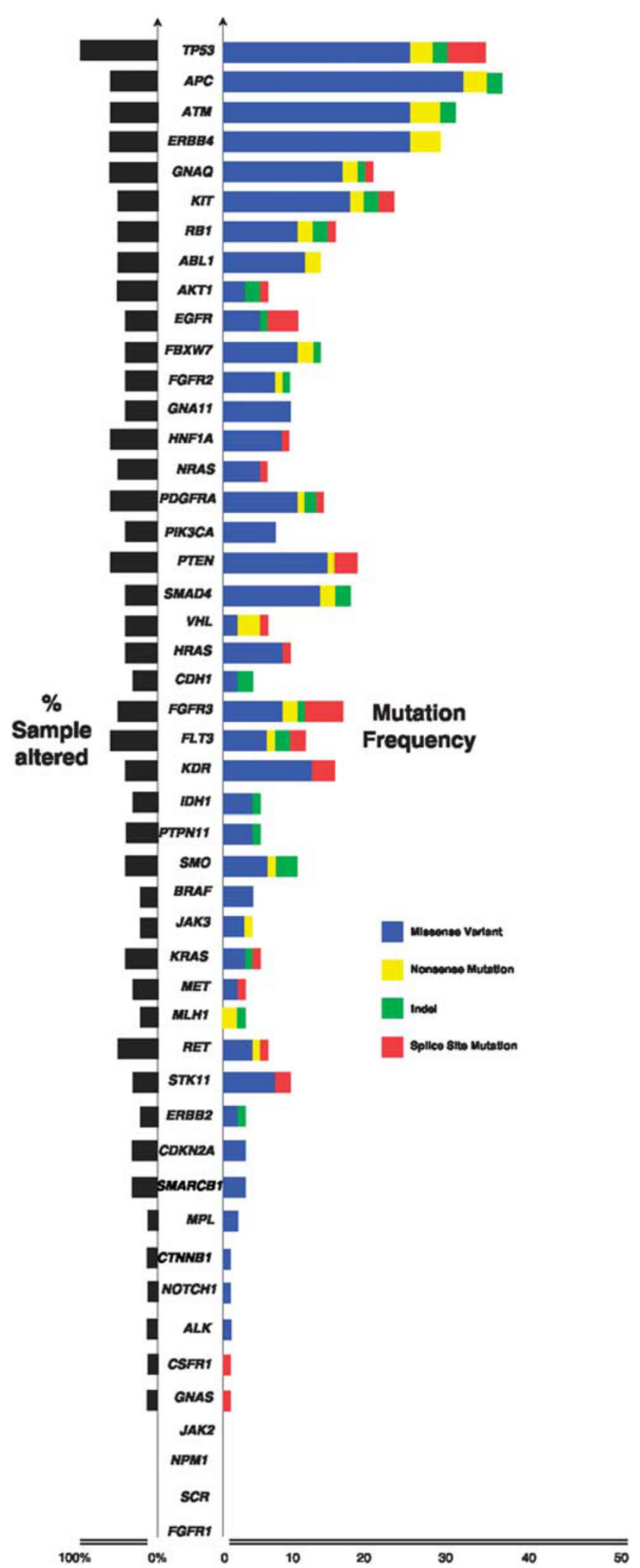

Figure 2 Genomic overview of mutations detected by targeted gene sequencing of 48 cancer-associated genes in 10 high-risk cases of non-metastatic HNcSCC. Missense variants (meeting at least one of-MutSig significance, FATHMM significance or known COSMIC mutation), nonsense mutations, insertions/deletions and splice variants illustrated in the right-hand plot. Lefthand plot demonstrates the percentage of samples with alterations detected in each given gene. 
Table 2 All functionally validated or likely significant mutations in tumor-suppressor genes

\begin{tabular}{|c|c|c|c|c|c|c|}
\hline $\begin{array}{l}\text { Case } \\
\text { number }\end{array}$ & $\begin{array}{l}\text { Gene } \\
\text { symbol }\end{array}$ & Mutation & COSMIC ID & Location & Evidence in other cancers & \\
\hline 1 & TP53 & c. $273 \mathrm{G}>\mathrm{A}, \mathrm{p} . \mathrm{W} 91^{*}$ & COSM44492 & $\begin{array}{l}\text { Premature truncation within proline-rich } \\
\text { domain, loss of DNA-binding domain (DBD) }\end{array}$ & $\begin{array}{l}\text { Biliary tract, breast, endometrium, esophagus } \\
\text { (carcinoma), lung (NSCLC-adenocarcinoma) }\end{array}$ & \\
\hline 1 & TP53 & c. $880 \mathrm{G}>\mathrm{T}, \mathrm{p}$ E294* & COSM10856 & Premature truncation & $\begin{array}{l}\text { Colorectal, breast, endometrium, lymphoid } \\
\text { (DLBCL) }\end{array}$ & \\
\hline 1 & $A P C$ & c.2752_2753delGA, p.R919Kfs*4 & N/A & $\begin{array}{l}\text { Frameshift deletion with Armadillo- } \\
\text { associated region, predicted premature } \\
\text { truncation with loss of downstream } \\
\text { functional domains }\end{array}$ & N/A & \\
\hline 1 & $A T M$ & c. $3991 \mathrm{C}>\mathrm{T}$, p. Q1331* & N/A & $\begin{array}{l}\text { Premature truncation, loss of downstream } \\
\text { functional domains }\end{array}$ & N/A & \\
\hline 1 & $V H L$ & c. $394 \mathrm{C}>\mathrm{T}$, p. Q132* & COSM14356 & $\begin{array}{l}\text { Premature truncation within VHL beta- } \\
\text { domain responsible for complex formation }\end{array}$ & $\begin{array}{l}\text { Kidney (clear cell), pancreas, soft tissue } \\
\text { (disseminated cerebellar hemangioblastoma) }\end{array}$ & \\
\hline 1 & PTEN & c. $49 \mathrm{C}>\mathrm{T}, \mathrm{p} . \mathrm{Q} 17^{*}$ & COSM5153 & $\begin{array}{l}\text { Premature truncation, loss of downstream } \\
\text { functional domains }\end{array}$ & $\begin{array}{l}\text { Breast, endometrium, lymphoid, lung } \\
\text { (adenocarcinoma) }\end{array}$ & \\
\hline 3 & TP53 & c. $655 \mathrm{C}>\mathrm{T}, \mathrm{p} . \mathrm{P} 219 \mathrm{~S}$ & COSM44076 & Missense mutation within DBD & $\begin{array}{l}\text { Esophagus (SCC), lymphoid (T-cell), } \\
\text { aerodigestive tract (HNSCC), leiomyosarcoma, } \\
\text { tumor growth in cell culture models }\end{array}$ & \\
\hline 3 & $A P C$ & c. $3982 \mathrm{C}>\mathrm{T}$, p. Q1328* & COSM18859 & $\begin{array}{l}\text { Premature truncation, loss of downstream } \\
\text { Ctnnb1-binding sites }\end{array}$ & Colorectal, endometrial & \\
\hline 3 & $A P C$ & c.4034delA, p. E1345Dfs*70 & N/A & $\begin{array}{l}\text { Frameshift deletion, leading to premature } \\
\text { truncation and loss of downstream Ctnnb1- } \\
\text { binding sites }\end{array}$ & N/A & \\
\hline 3 & SMAD4 & c. $364 \mathrm{~A}>\mathrm{T}, \mathrm{p} . \mathrm{K} 122^{*}$ & N/A & $\begin{array}{l}\text { Premature truncation within MAD homology } \\
\text { (MH1) domain involved in nuclear import } \\
\text { and protein interactions }\end{array}$ & N/A & 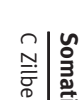 \\
\hline 3 & SMAD4 & c.533delC, p.S178* & N/A & $\begin{array}{l}\text { Frameshift deletion, leading to premature } \\
\text { truncation, loss of downstream MH2 } \\
\text { functional domain }\end{array}$ & N/A & 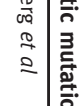 \\
\hline 3 & $R B 1$ & c. $1735 \mathrm{C}>\mathrm{T}$, p. R579* & COSM892 & $\begin{array}{l}\text { Premature truncation, loss of downstream } \\
\text { functional domains }\end{array}$ & $\begin{array}{l}\text { Biliary tract, lung (SCLC), melanoma, } \\
\text { retinoblastoma, lymphoid (COSMIC unknown), } \\
\text { BCC }\end{array}$ & \\
\hline 1,3 & $A T M$ & c. $7327 \mathrm{C}>\mathrm{T}, \mathrm{p} . \mathrm{R} 2443^{*}$ & COSM21678 & $\begin{array}{l}\text { Premature truncation within focal adhesion } \\
\text { targeting (FAT) domain }\end{array}$ & $\begin{array}{l}\text { Adrenal cortex (adenoma), lymphoid (mantle } \\
\text { cell) }\end{array}$ & 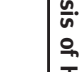 \\
\hline 5 & TP53 & c. $818 \mathrm{G}>\mathrm{A}, \mathrm{p} . \mathrm{R} 273 \mathrm{H}$ & COSM10660 & Missense mutation within DBD & $\begin{array}{l}\text { Biliary tract, bone (chondrosarcoma, Ewing's } \\
\text { sarcoma, osteosarcoma), increased cell migration }\end{array}$ & 甹 \\
\hline 5 & TP53 & c. $880 \mathrm{G}>\mathrm{T}, \mathrm{p} . \mathrm{R} 280 \mathrm{~K}$ & COSM330620 & Missense mutation within DBD & $\begin{array}{l}\text { Breast, colorectal, ovarian, hematopoietic } \\
\text { (primary plasma cell leukemia), increased } \\
\text { activation novel targets }\end{array}$ & \\
\hline 5 & $A T M$ & c. $1063 \mathrm{C}>\mathrm{T}, \mathrm{p} . \mathrm{Q} 355^{*}$ & N/A & $\begin{array}{l}\text { Premature truncation, loss of downstream } \\
\text { functional domains }\end{array}$ & N/A & \\
\hline 5 & $A T M$ & c.2617delG, p.G873Efs*7 & N/A & $\begin{array}{l}\text { Frameshift deletion, leading to premature } \\
\text { truncation with loss of downstream } \\
\text { functional domains }\end{array}$ & N/A & \\
\hline 5 & $A T M$ & c.5066delA, p.Q1689Hfs*25 & N/A & $\begin{array}{l}\text { Frameshift deletion, leading to premature } \\
\text { truncation, loss of downstream functional } \\
\text { domains }\end{array}$ & N/A & \\
\hline 5 & MLH1 & c.1219C> T, p. Q407* & N/A & $\begin{array}{l}\text { Premature truncation, loss C-terminal } \\
\text { dimerization domain }\end{array}$ & N/A & \\
\hline 5 & MLH1 & c.1264_1268delGGCAG, p.R423* & N/A & & N/A & \\
\hline
\end{tabular}


Table 2 (Continued)

\begin{tabular}{|c|c|c|c|c|c|}
\hline $\begin{array}{l}\text { Case } \\
\text { number }\end{array}$ & $\begin{array}{l}\text { Gene } \\
\text { symbol }\end{array}$ & Mutation & COSMIC ID & Location & Evidence in other cancers \\
\hline & & & & $\begin{array}{l}\text { Frameshift deletion, leading to premature } \\
\text { truncation, loss downstream functional } \\
\text { domains }\end{array}$ & \\
\hline 5 & SMAD4 & c. $1342 \mathrm{C}>\mathrm{T}, \mathrm{p} . \mathrm{Q} 448^{*}$ & N/A & $\begin{array}{l}\text { Premature truncation within } \mathrm{MH} 2 \text { domain } \\
\text { involved in protein interactions }\end{array}$ & N/A \\
\hline 5 & $V H L$ & c.583C > T, p. Q195* & N/A & $\begin{array}{l}\text { Premature truncation within VHL box } \\
\text { domain }\end{array}$ & N/A \\
\hline 5 & $C D H 1$ & c. $1118 \mathrm{C}>\mathrm{T}$, p. P373L & N/A & $\begin{array}{l}\text { Missense mutation upstream from cadherin } 3 \\
\text { domain }\end{array}$ & $\begin{array}{l}\text { Reduced interaction with EGFR, increased } \\
\text { motility cell culture }\end{array}$ \\
\hline 5 & $C D H 1$ & c.1240delA, p.T414Pfs*3 & N/A & $\begin{array}{l}\text { Frameshift deletion within cadherin domain } \\
3 \text {, leading to premature truncation }\end{array}$ & N/A \\
\hline 5 & $F B X W 7$ & c. $1338 \mathrm{G}>\mathrm{A}, \mathrm{p} . \mathrm{W} 446^{*}$ & COSM22986 & $\begin{array}{l}\text { Premature truncation within WD2 repeat } \\
\text { domain }\end{array}$ & Colorectal \\
\hline 5 & $F B X W 7$ & c.1469delC, p.T490Kfs*8 & N/A & $\begin{array}{l}\text { Frameshift deletion, predicted premature } \\
\text { truncation, loss of downstream functional } \\
\text { domains }\end{array}$ & N/A \\
\hline 5 & $R B 1$ & c.1090G > T, p. E364* & N/A & $\begin{array}{l}\text { Premature truncation, loss of downstream } \\
\text { functional domains }\end{array}$ & N/A \\
\hline 5 & $R B 1$ & c.1811_1814+11delATATGTAAGCAAAAT & N/A & $\begin{array}{l}\text { Frameshift deletion within Rb-associated } \\
\text { protein B domain, predicted premature } \\
\text { truncation }\end{array}$ & N/A \\
\hline 2 & TP53 & $\begin{array}{l}\text { c.502_513delCACATGACGGAG, p. } \\
\text { H168_E171delinsdel }\end{array}$ & N/A & Frameshift deletion within DBD & N/A \\
\hline 2 & $C D H 1$ & c.349delA, p.N117Ifs*98 & N/A & $\begin{array}{l}\text { Frameshift deletion within cadherin } 2 \\
\text { domain, loss of downstream functional } \\
\text { domains }\end{array}$ & N/A \\
\hline 4 & TP53 & c. $310 \mathrm{C}>\mathrm{T}, \mathrm{p} . \mathrm{Q} 104^{*}$ & COSM10886 & Premature truncation within DBD & $\begin{array}{l}\text { Biliary tract, breast, esophagus (carcinoma), } \\
\text { hematopoietic (AML), lung (NSCLC- } \\
\text { adenocarcinoma) }\end{array}$ \\
\hline 4 & $V H L$ & c. $337 \mathrm{C}>\mathrm{T}$, p. R113* & COSM30228 & $\begin{array}{l}\text { Premature truncation within VHL beta- } \\
\text { domain responsible for complex formation }\end{array}$ & Kidney (clear cell) \\
\hline 4 & $F B X W 7$ & c. $1458 \mathrm{G}>\mathrm{A}, \mathrm{p} . \mathrm{W} 486^{*}$ & COSM87016 & $\begin{array}{l}\text { Premature truncation within WD3 } \\
\text { (tryptophan-aspartic acid) repeat domain }\end{array}$ & Colorectal, tumor growth cell culture \\
\hline 6 & TP53 & c. $844 \mathrm{C}>\mathrm{T}, \mathrm{p} . \mathrm{R} 282 \mathrm{~W}$ & COSM10704 & Missense mutation within DBD & cSCC, BCC, tumor growth mouse models \\
\hline 7 & TP53 & c. $746 \mathrm{G}>\mathrm{T}$, p. R249M & COSM43871 & Missense mutation within DBD & $\begin{array}{l}\text { Breast, colorectal, liver, bone (Ewing sarcoma), } \\
\text { CNS (glioma), lymphoid (CLL), hematopoietic } \\
\text { (myelodysplasia) }\end{array}$ \\
\hline 7 & TP53 & c.617delT, p.L206Wfs*41 & N/A & Premature truncation within DBD & N/A \\
\hline 7 & $A P C$ & c. $3340 \mathrm{C}>\mathrm{T}, \mathrm{p} . \mathrm{R} 1114^{*}$ & COSM13125 & $\begin{array}{l}\text { Premature truncation within unstructured } \\
\text { region of APC, leading to loss of downstream } \\
\text { Ctnnb1-binding sites }\end{array}$ & Colorectal, endometrial \\
\hline 9 & TP53 & c. $1010 \mathrm{G}>\mathrm{A}$, p. R337H & COSM43882 & $\begin{array}{l}\text { Missense mutation within tetramerization } \\
\text { domain }\end{array}$ & $\begin{array}{l}\text { Adrenal (carcinoma, pheochromocytoma), cervix } \\
\text { colorectal, liver, disrupted tetramer formation in } \\
\text { cell culture }\end{array}$ \\
\hline 9 & $A P C$ & c.4549C > T, p. Q1517* & N/A & $\begin{array}{l}\text { Premature truncation, leading to downstream } \\
\text { loss of Ctnnb1-binding sites }\end{array}$ & Loss of beta-catenin regulation \\
\hline 9 & MLH1 & c.1276C> T, p. Q426* & N/A & $\begin{array}{l}\text { Premature truncation loss C-terminal } \\
\text { dimerization domain }\end{array}$ & N/A \\
\hline 9 & SMAD4 & c.1186delG, p.D396Mfs*19 & N/A & $\begin{array}{l}\text { Frameshift deletion within } \mathrm{MH} 2 \text { domain, } \\
\text { leading to premature truncation }\end{array}$ & N/A \\
\hline 10 & TP53 & c. $832 \mathrm{C}>\mathrm{T}$, p. $\mathrm{P} 278 \mathrm{~S}$ & COSM10939 & Missense mutation within DBD & Breast, tumor growth xenograft models \\
\hline
\end{tabular}




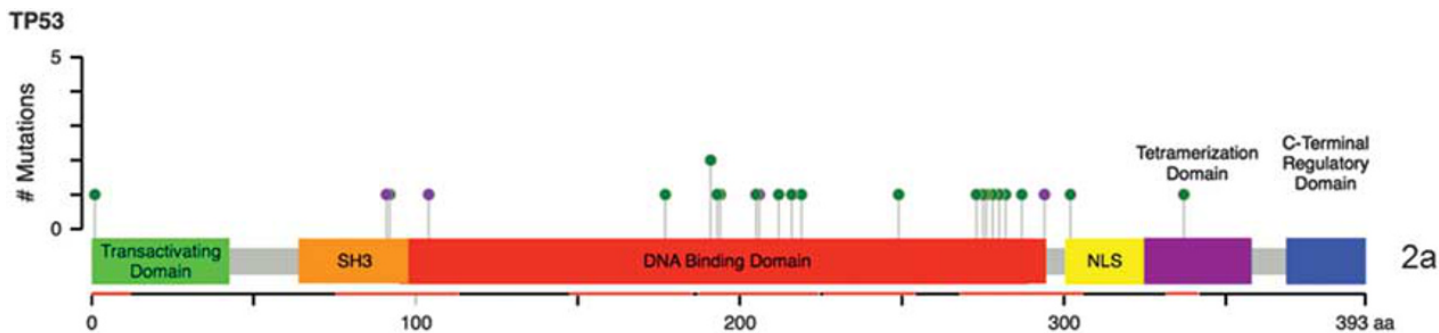

APC

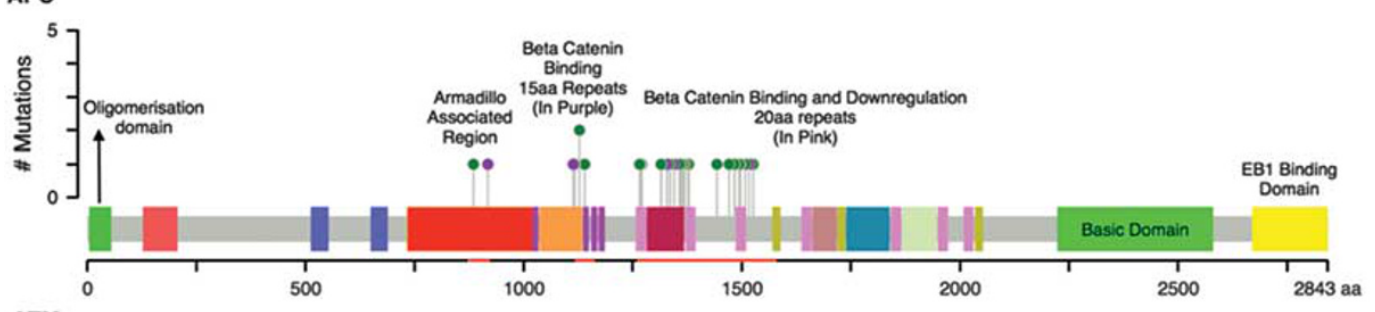

ATM

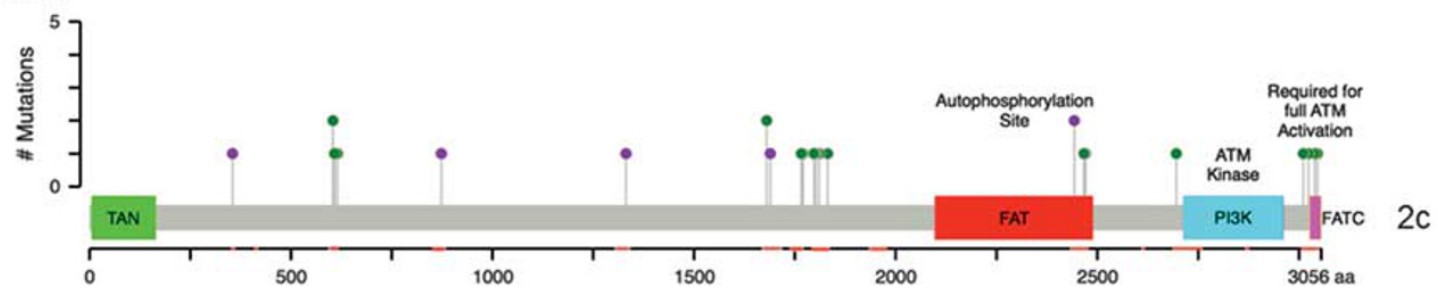

Figure 3 Visual representation of the location of the detected missense variants, nonsense mutations, insertions and deletions in 3a. TP53, 3b. APC and 3c, ATM. Green-missense variants; Purple-nonsense mutations and gene deletions. Red highlights $X$ axis represent areas covered by TruSeq amplicon panel.

generation of head and neck cutaneous squamous cell carcinoma in mice. ${ }^{20}$

Loss-of-function mutations in $V H L$ were identified in three cases, including R113* and Q132*; both of which are well characterized in renal cell carcinoma. Loss-of-function mutations in PTEN gene have been associated with disease progression and resistance to radiotherapy in head and neck and pulmonary squamous cell carcinoma. ${ }^{21,22} \mathrm{~A}$ validated loss-offunction mutation in PTEN, Q17*, was observed in only a single patient. We noted three functionally significant mutations in FBXW7 including W446* and $\mathrm{W}_{486}{ }^{*}$ in two patients, leading to premature truncation of the WD2 repeat domain. Both are well described in colorectal carcinoma (Table 2).

CDKN2A mutations are well characterized in cutaneous squamous cell carcinoma and in melanoma. Three cases had mutations in this gene, two of these are the known truncating variants within the ankyrin-repeat containing domain, R58* and E61*, described in cutaneous squamous cell carcinoma and melanoma. ${ }^{23}$

Known gain-of-function mutations in oncogenes were present in $60 \%$ of samples. A total of 12 functionally relevant oncogenic gain-of-function mutations were present across 10 genes (Table 3). Mutations in receptor tyrosine kinases resulting in constitutive tyrosine kinase activity and elevated downstream signaling were seen in this cohort. Two mutations, HRAS G13D and BRAF G464R, have been described in a metastatic cutaneous squamous cell carcinoma cohort. ${ }^{24}$ The BRAF mutation, and alterations in KDR Q472H, KIT T670I and GNAQ R183Q have been previously detected in cutaneous and uveal melanoma samples. ${ }^{25,26}$ EGFR A864T is a rare activating variant ${ }^{27}$ that was also seen in this cohort. An activating JAK3 A572T mutation, previously detected in T-cell acute lymphoblastic leukemia at a location shown to promote hematological malignancy in mice was identified. Two alterations in PTPN11 (D61Y, T73I), characteristically found in hematological malignancies, ${ }^{28}$ were also identified in two patients.

\section{Association with Clinicopathologic Characteristics}

Novel somatic mutations in MLH1 (Q407*, Q426*, R423*) leading to premature truncation and loss of the C-terminal dimerization domain were seen in two patients, both younger than 45 years of age.

Somatic missense mutations in the receptor tyrosine kinase FGFR2 were exclusively seen in patients with histologic evidence of perineural invasion. Of these, FGFR2 N549K and FGFR2 M536I are validated activating mutations, conferring constitutive tyrosine kinase activation and facilitating tumorigenesis in cell culture. ${ }^{29}$ FGFR2 N549K is well documented in endometrial carcinoma. ${ }^{30}$ In addition, two novel mutations, FGFR2 A380D and 
Table 3 All functionally validated mutations in oncogenes

\begin{tabular}{|c|c|c|c|c|c|}
\hline $\begin{array}{l}\text { Case } \\
\text { number }\end{array}$ & Gene symbol & Mutation & COSMIC ID & Location and functional effect & Evidence \\
\hline 3 & EGFR & $\begin{array}{l}\text { c. } 2590 \mathrm{G}>\mathrm{A}, \mathrm{p} . \\
\text { A864T }\end{array}$ & COSM13197 & $\begin{array}{l}\text { Missense mutation within protein } \\
\text { tyrosine kinase (PTK) domain }\end{array}$ & $\begin{array}{l}\text { NSCLC (large cell), biliary tract, adrenal (carcinoma), Increased Tyr } \\
\text { kinase activity in cell culture }\end{array}$ \\
\hline 5 & KIT & $\begin{array}{l}\text { c. } 2009 \mathrm{C}>\mathrm{T}, \mathrm{p} . \\
\text { T670I }\end{array}$ & COSM12708 & $\begin{array}{l}\text { Missense mutation within ATP-binding } \\
\text { pocket }\end{array}$ & $\begin{array}{l}\text { GIST, melanoma, constitutive Tyr kinase phosphorylation cell culture, } \\
\text { gain-of-function }\end{array}$ \\
\hline 1 & $K D R$ & $\begin{array}{l}\text { c. } 1416 \mathrm{~A}>\mathrm{T}, \mathrm{p} . \\
\mathrm{Q} 472 \mathrm{H}\end{array}$ & COSM149673 & $\begin{array}{l}\text { Missense mutation; does not lie in known } \\
\text { functional domain }\end{array}$ & $\begin{array}{l}\text { Neuroblastoma, GIST, bone, melanoma, lymphoid (DLBCL), colorectal, } \\
\text { rhabdomyosarcoma, increased Tyr kinase activity cell culture, increased } \\
\text { angiogenesis tumor samples }\end{array}$ \\
\hline 5 & $B R A F$ & $\begin{array}{l}\text { c. } 1390 \mathrm{G}>\mathrm{A}, \mathrm{p} \text {. } \\
\text { G464R }\end{array}$ & COSM1448615 & Missense mutation within PTK domain & $\begin{array}{l}<1 \% \text { melanomas, cSCC, increased Tyr kinase activity in cell culture, } \\
\text { gain-of-function }\end{array}$ \\
\hline 3 & FGFR2 & $\begin{array}{l}\text { c. } 1608 \mathrm{G}>\mathrm{A}, \mathrm{p} \text {. } \\
\text { M536I }\end{array}$ & N/A & Missense mutations within PTK domain & $\begin{array}{l}\text { Increased kinase activity and enhanced cell proliferation in the presence } \\
\text { of ligand in culture }\end{array}$ \\
\hline 3 & FGFR2 & $\begin{array}{l}\text { c. } 1646 \mathrm{~T}>\mathrm{A}, \mathrm{p} . \\
\text { N549K }\end{array}$ & N/A & Missense mutations within PTK domain & $\begin{array}{l}\text { Confers a gain-of-function to the Fgfr2 protein, resulting in oncogenic } \\
\text { transformation in cell-based studies }\end{array}$ \\
\hline 7 & $G N A Q$ & $\begin{array}{l}\text { c. } 548 \mathrm{G}>\mathrm{A}, \mathrm{p} . \\
\text { R183Q }\end{array}$ & COSM52975 & $\begin{array}{l}\text { Missense mutation within nucleotide } \\
\text { binding region }\end{array}$ & $\begin{array}{l}\text { Uveal melanoma, colorectal, reduced GTPase activity in cell culture, } \\
\text { increased downstream signaling, gain-of-function }\end{array}$ \\
\hline 1 & HRAS & c. $38 \mathrm{G}>\mathrm{A}, \mathrm{p} . \mathrm{G} 13 \mathrm{D}$ & COSM490 & $\begin{array}{l}\text { Missense mutation within the GTP } \\
\text { nucleotide binding region }\end{array}$ & $\begin{array}{l}\text { Inhibits GTPase activity leading to increased activation of downstream } \\
\text { signaling in the absence of activation }\end{array}$ \\
\hline 9 & $J A K 3$ & $\begin{array}{l}\text { c. } 1714 \mathrm{G}>\mathrm{A}, \mathrm{p} . \\
\text { A572T }\end{array}$ & COSM327318 & Missense mutation within PTK domain & $\begin{array}{l}\text { Lymphoid (T-ALL), increased Tyr kinase activity in cell culture, gain-of- } \\
\text { function }\end{array}$ \\
\hline 9 & PTPN11 & $\begin{array}{l}\text { c. } 181 \mathrm{G}>\mathrm{T}, \mathrm{p} . \\
\text { D } 61 \mathrm{Y}\end{array}$ & COSM13011 & $\begin{array}{l}\text { Missense mutation within Src homology } 2 \\
\text { (SH2) domain }\end{array}$ & $\begin{array}{l}\text { Hematopoietic (AML, ALL), neuroblastoma, Increased Tyr phosphatase } \\
\text { activity in cell culture, gain-of-function }\end{array}$ \\
\hline 1 & PTPN11 & c. $218 \mathrm{C}>\mathrm{T}, \mathrm{p} . \mathrm{T} 73 \mathrm{I}$ & COSM13019 & Missense mutation within $\mathrm{SH} 2$ & $\begin{array}{l}\text { Hematopoietic (myelodysplastic syndrome, CML, ALL, AML), increased } \\
\text { Tyr phosphatase activity in cell culture, gain-of-function }\end{array}$ \\
\hline 4 & $S M O$ & $\begin{array}{l}\text { c. } 1234 \mathrm{C}>\mathrm{T}, \mathrm{p} \text {. } \\
\mathrm{L} 412 \mathrm{~F}\end{array}$ & COSM216037 & $\begin{array}{l}\text { Missense mutation within pivot region } \\
\text { transmembrane (TM) helix } 5\end{array}$ & $\begin{array}{l}\text { Bone (ameloblastoma), constitutive activation of Hedgehog }(\mathrm{HH}) \\
\text { signaling in cell culture, gain-of-function }\end{array}$ \\
\hline
\end{tabular}


Table 4 All functionally validated mutations in oncogenes susceptible to currently approved or preclinical small molecule targeted therapies

\begin{tabular}{|c|c|c|c|c|c|c|}
\hline $\begin{array}{l}\text { Case } \\
\text { number }\end{array}$ & $\begin{array}{l}\text { Gene } \\
\text { symbol }\end{array}$ & Mutation & Functional effect & Cellular pathway & Drug susceptibility & Evidence/use \\
\hline 3 & EGFR & $\begin{array}{l}\text { c. } 2590 \mathrm{G}>\mathrm{A}, \mathrm{p} . \\
\text { A } 864 \mathrm{~T}\end{array}$ & $\begin{array}{l}\text { Gain-of-function; constitutive kinase } \\
\text { activity }\end{array}$ & MAPK & Erlotinib, Gefitinib & Non-small cell lung cancer \\
\hline 7 & KIT & $\begin{array}{l}\text { c. } 2009 \mathrm{C}>\mathrm{T}, \mathrm{p} . \\
\text { T670I }\end{array}$ & $\begin{array}{l}\text { Gain-of-function, leads to constitutive } \\
\text { phosphorylation of KIT }\end{array}$ & MAPK & Sorafenib, resistant to Imatinib & $\begin{array}{l}\text { Kidney, liver, GIST } \\
\text { (Sorafenib) melanoma } \\
\text { (preclinical) }\end{array}$ \\
\hline 1 & $K D R$ & $\begin{array}{l}\text { c. } 1416 \mathrm{~A}>\mathrm{T}, \mathrm{p} \text {. } \\
\text { Q472H }\end{array}$ & $\begin{array}{l}\text { Gain-of-function; increased } \\
\text { phosphorylation }\end{array}$ & Angiogenesis & VEGFR inhibitors & $\begin{array}{l}\text { Trials (NSCLC), preclinical } \\
\text { (melanoma) }\end{array}$ \\
\hline 3 & FGFR2 & $\begin{array}{l}\text { c. } 1646 \mathrm{~T}>\mathrm{A}, \mathrm{p} . \\
\text { I549K }\end{array}$ & $\begin{array}{l}\text { Gain-of-function; constitutive kinase } \\
\text { activity }\end{array}$ & MAPK & $\begin{array}{l}\text { Ponatinib (resistance to Dovitinib, } \\
\text { PD173074); combination mTOR inhibitor } \\
\text { (Ridaforolimus) }\end{array}$ & $\begin{array}{l}\text { Preclinical (endometrial } \\
\text { cancer cell lines, BaF3 cell } \\
\text { lines) }\end{array}$ \\
\hline 3 & FGFR2 & $\begin{array}{l}\text { c. } 1608 \mathrm{G}>\mathrm{A}, \mathrm{p} \text {. } \\
\text { M536I }\end{array}$ & $\begin{array}{l}\text { Gain-of-function; constitutive kinase } \\
\text { activity }\end{array}$ & MAPK & $\begin{array}{l}\text { Ponatinib (decreased response to } \\
\text { Dovitinib, PD173074) }\end{array}$ & $\begin{array}{l}\text { Preclinical (endometrial } \\
\text { cancer; BaF3 lines) }\end{array}$ \\
\hline 6 & $G N A Q$ & $\begin{array}{l}\text { c. } 548 G>\text { A, p. } \\
\text { R183Q }\end{array}$ & $\begin{array}{l}\text { Loss-of-function; reduced GTPase } \\
\text { activity }\end{array}$ & PI3K; mTOR; PI3K & Combined PKC, MEK inhibitors & Preclinical \\
\hline 1 & HRAS & $\begin{array}{l}\text { c. } 38 G>A, p \\
\text { G13D }\end{array}$ & $\begin{array}{l}\text { Loss-of-function; inhibition GTPase } \\
\text { activity; increased downstream } \\
\text { signaling }\end{array}$ & MAPK; PI3K & $\begin{array}{l}\text { Combined MET, MEK inhibitors } \\
\text { (resistance to MET inhibition); Ras } \\
\text { inhibitors }\end{array}$ & Preclinical \\
\hline 9 & PTPN11 & $\begin{array}{l}\text { c. } 181 \mathrm{G}>\mathrm{T}, \mathrm{p} . \\
\mathrm{D} 61 \mathrm{Y}\end{array}$ & $\begin{array}{l}\text { Gain-of-function; increased Tyr } \\
\text { phosphatase activity }\end{array}$ & MAPK & SHP2 inhibitor (actually shown in vivo) & Preclinical \\
\hline 1 & PTPN11 & $\begin{array}{l}\text { c. } 218 \mathrm{C}>\mathrm{T}, \mathrm{p} . \\
\text { T73I }\end{array}$ & $\begin{array}{l}\text { Gain-of-function; increased Tyr } \\
\text { phosphatase activity }\end{array}$ & MAPK & SHP2 inhibitor & Preclinical \\
\hline 4 & $S M O$ & $\begin{array}{l}\text { c. } 1234 \mathrm{C}>\mathrm{T}, \mathrm{p} \text {. } \\
\mathrm{L} 412 \mathrm{~F}\end{array}$ & $\begin{array}{l}\text { Gain-of-function; constitutive } \\
\text { (Hedgehog) HH signaling }\end{array}$ & Hedgehog & GLI inhibitors; Vismodegib resistance & Preclinical \\
\hline
\end{tabular}


D528N leading to changes within the transmembrane domain and protein tyrosine kinase domain, respectively, were also observed in this cohort. Of the four patients with evidence of perineural invasion, two had histologic evidence of lymphovascular invasion. There were no unique findings in this subgroup. In addition, there were no differences in mutations according to histologic differentiation.

NOTCH1 alterations were observed in one patient in this non-metastatic cohort using the targeted panel covering 43 commonly mutated amino-acid residues in positions 1562-1600 and 1674-1678 in the NOTCH1 protein. The NOTCH1 L1569P mutation occurs within a functionally significant negative regulatory region of the gene, however, the functional significance, if any, of this mutation has not been described in the literature.

\section{Somatic Mutations in HNcSCC and Targeted Therapies}

Table 4 lists 10 activating mutations in oncogenes, which demonstrate susceptibility to currently clinically available targeted therapeutics or to small molecule drugs undergoing clinical trials or preclinical investigations. In all, $60 \%$ of the cases demonstrated at least one alteration amenable to small molecule therapy, although recurrent alterations were rare. Of these, three cases showed mutually exclusive mutations (EGFR A864T, KIT T670I) with sensitivity to approved therapies (Erlotinib/Gefitinib -EGFR inhibitors, Sorafenib-broad tyrosine kinase inhibitor, respectively) that are used in other malignancies. One of the patients showed a KDR Q472H mutation, which is susceptible to the antiVEGF drug Bevacizumab that is approved for the treatment of metastatic cervical, colorectal, breast and renal cancers. ${ }^{31}$ HRAS G13D, which demonstrates in vitro susceptibility to combined MET and MEK inhibition, and to novel Ras inhibitors ${ }^{32,33}$ was seen in one patient, and was mutually exclusive to the patient with an EGFR activating mutation. The SMO L412F mutation, previously detected in basal cell carcinoma, shows resistance to Vismodegib, ${ }^{34}$ but may be susceptible to a new class of compounds, the GLI inhibitors that are under investigation for Hedgehog pathway blockade. ${ }^{34}$

\section{Discussion}

The current cohort of 10 patients is unique, in that it represents a group of high-risk head and neck cutaneous squamous cell carcinoma tumors with a median depth of invasion of $10.5 \mathrm{~mm}$ and without evidence of metastatic disease. In addition to confirming the presence of the typical UV signature of C/ $\mathrm{T}$ transitions and the high incidence of tumorsuppressor mutations including TP53, the analysis puts forth several findings. These include; presence of somatic mutations in MLH1 in young patients with cSCC, presence of FGFR2 mutations exclusively in patients with perineural invasion, lower incidence NOTCH1 mutations in this non-metastatic cohort as compared with metastatic cohorts described in the literature and the presence of actionable mutations with targeted therapeutic agents approved for other common malignancies in nearly $60 \%$ of cases.

TP53 loss-of-function is hypothesized to occur early in cutaneous squamous cell carcinoma pathogenesis. ${ }^{23}$ The loss of heterozygosity is associated with a sharp increase in mutation burden. ${ }^{23}$ Further evidence supporting early mutation of TP53 in skin comes from studies of physiologically normal, sun-exposed skin with clones of TP53 mutant cells. ${ }^{35,36}$ Of the 11 functionally validated TP53 mutations in the current cohort, eight affected the DNA-binding domain of the gene. The location of the gene mutation may have predictive roles in disease prognosis. Although this has not been explored in cutaneous SCC; TP53 mutations within the DNA-binding domain have been found to be an independent predictor of poor disease specific survival in oral squamous cell carcinoma. ${ }^{37}$

Mutations in $A T M$ were detected in $60 \%$ of tumor samples. ATM is an integral factor in the DNA damage response pathway, orchestrating signaling following UV damage. ${ }^{38}$ In vitro changes to phosphorylated ATM localization (and hence signaling) have been identified in normal, sun-exposed, premalignant and cutaneous squamous cell carcinoma cell lines. ${ }^{39}$ Interestingly, the two youngest patients ( $<45$ years) showed functionally significant somatic mutations in both ATM and MLH1. These patients also showed relatively high total mutation burdens (198 and 502). Familial MLH1 alterations are characteristic of hereditary non-polyposis colon cancer syndrome. Sebaceous carcinoma is a wellknown component of the Muir-Torre syndrome, however, there is no information regarding cutaneous squamous cell carcinoma as a component of hereditary non-polyposis colon cancer syndrome. ${ }^{40}$ The role of somatic mutations in MLH1 and its association with earlier age of onset of cutaneous squamous cell carcinoma is not well studied.

FGFR2 is a receptor tyrosine kinase mediating cell division, growth and differentiation signaling. Immunohistochemical overexpression of FGFR2 has been described to be associated with perineural invasion, ${ }^{41}$ advanced tumor stage and shorter survival in patients receiving neoadjuvant chemotherapy for rectal cancers. ${ }^{42}$ Amplification of FGFR2 in gastric cancer is related to regional lymph node metastases and subsequent poor prognosis. ${ }^{43}$ Interestingly, FGFR2 mutations in our patient cohort were seen only in those with histologic evidence of perineural invasion. However, the role of missense mutations in the protein tyrosine kinase domain of FGFR2 and its role in perineural invasion is not documented.

In the current non-metastatic cohort, we detected a single example of a mutation in the NOTCH1 gene. The functional significance of this alteration appears 
to be limited. This contrasts with information obtained from the DNA analysis of metastatic tumors, which demonstrated NOTCH1 mutations in $69 \%$ of the cohort. $^{24}$ In cutaneous squamous cell carcinomas from immunocompromised patients NOTCH1/2 was altered in $89 \%$ of samples, ${ }^{44}$ and Pickering et $a l^{14}$ described $30 \%$ prevalence of NOTCH1 inactivating mutations. A direct comparison of the various studies evaluating NOTCH changes in cutaneous squamous cell carcinoma is primarily limited by the wide variety of the testing methods used such as whole-exome sequencing and capture panel analysis. The Illumina TruSeq Amplicon Cancer Panel ${ }^{\circledR}$ used in the current study covers approximately $35 \mathrm{~kb}$ (exons 26 AA 1562-1600; exon 27 AA 1674-1678) of NOTCH1. In addition, combinations of primary and metastatic cohorts have been used in the literature without further information regarding the distribution of $\mathrm{NOTCH}$ mutations in primary or metastatic tissues. Also, the details of the quality control checks while using formalin-fixed paraffin-embedded samples and the functional significance of the various alterations described in these studies are not readily available. For instance, the incidence of NOTCH1 mutations drops from $69 \%$ to only $24 \%$ in the metastatic cohort described by $\mathrm{Li}$ et $a l^{24}$ when only functionally significant mutations are considered. NOTCH1 has multifaceted roles in carcinogenesis. It has been proposed that loss of NOTCH1 is not an initiator of disease, but acts more as a cancer-promoting event. ${ }^{45}$ Thus, the role of this gene in regional and distant progression of head and neck cutaneous squamous cell carcinoma bears further investigation in well-designed cohorts using comprehensive DNA and expression analysis. This is particularly significant currently, as therapeutic targets modulating NOTCH activity are under development. ${ }^{46}$

There is an unmet need for effective medical treatment of invasive and metastatic cutaneous squamous cell carcinoma. It has been hypothesized that this disease is largely tumor-suppressor driven in etiology, ${ }^{14}$ which, combined with the consistently reported high mutation burden of cSCC, has been a barrier to the development of targeted therapies. Our findings indicate mutations in oncogenes such as $E G F R, K I T, K D R, G N A Q$ and $E R B B 4$, although these were largely mutually exclusive non-recurrent events in this cohort. Similar findings have also been described by Li et al and Al-Rohil et al. ${ }^{24,47}$ Ten somatic mutations identified in six patients in this cohort may potentially be susceptible to currently approved therapies or to small molecule drugs under development. This finding merits further investigations, particularly as a phase two study evaluating use of gefitinib in aggressive cutaneous squamous cell carcinoma demonstrated favorable survival outcomes. ${ }^{48}$ Cetuximab has been trialed in a small cohort of patients, achieving a $69 \%$ disease control rate after 6 weeks of treatment including eight partial and two complete responses. ${ }^{49}$
The targeted panel used in this study is highly biased toward receptor tyrosine kinase genes involved in the MAPK, PI3K and mTOR signaling pathways, and nearly $90 \%$ of cases contained alterations in genes belonging to PI3K/mTOR pathway. Reduced rates of cutaneous squamous cell carcinoma development have been observed in organ transplant recipients receiving mTOR inhibitors. ${ }^{50}$ Al-Rohil et $a l^{47}$ have also recently described a clinical response in a patient with cSCC with PIK3CA P471L mutation, treated with the mTOR inhibitor temsirolimus. Although five of our patients showed PIK3CA mutations, no known targetable mutations were identified in this gene.

The chief limitations of this study include the small cohort size, use of formalin-fixed paraffinembedded samples and use of a targeted panel. Although cutaneous squamous cell carcinoma is a common malignancy, locally advanced high-risk tumors that have not developed metastases at a minimum follow-up of 2 years are extremely rare, particularly if complete clinicopathologic data and follow-up are also required. Furthermore, stringent quality control checks were applied to ensure that only those cases with high-quality DNA were included in analysis. Extraction of high-quality DNA from archival material is inherently difficult, leading to further shrinkage of the cohort. The TruSeq Amplification Cancer Panel is suitable for the fragmented DNA obtained from formalin-fixed paraffin-embedded samples and allows for cost effective data analysis in a clinically relevant time frame that can be replicated in other centers with DNA-sequencing facilities. Furthermore, our data shows several of the alterations identified by more comprehensive techniques requiring fresh tissue. ${ }^{14,44}$ Thus, we believe that our findings are likely to be reproduced in other study cohorts of non-metastatic, high-risk disease.

In conclusion, we have performed targeted sequencing of 48 cancer-associated genes on a unique cohort of 10 high-risk, non-metastatic head and neck cutaneous squamous cell carcinoma cases to a mean coverage of 6907 . Our results confirm the presence of a UV DNA damage signature, a high mutation burden and the predominance of TP53 mutations in disease pathogenesis. In addition, we describe several novel findings, including somatic mutations in MLH1 in younger patients with head and neck cutaneous squamous cell carcinoma, FGFR2 mutations in patients with perineural invasion and a low incidence of NOTCH1 mutations in this cohort, all of which open further avenues of study. Our data also indicate the presence of targetable mutations in a significant proportion of tumors, suggesting further treatment options for head and neck cutaneous squamous cell carcinoma, an under-researched disease with significant morbidity and mortality in the fair skinned population. 


\section{Acknowledgments}

We gratefully acknowledge the philanthropic financial support from ICAP, the O'Sullivan family, the Tag family foundation and David Paradice for their generosity and funding assistance. The preliminary results of this study have been presented at the United States and Canadian Academy of pathologists meeting in San Antonio, USA in March 2017 and at the Australia and New Zealand Head and Neck Cancer Society $18^{\text {th }}$ Annual Scientific Meeting in October 2016 Auckland, New Zealand.

\section{Disclosure/conflict of interest}

The authors declare no conflict of interest.

\section{References}

1 Gurudutt VV, Genden EM. Cutaneous squamous cell carcinoma of the head and neck. J Skin Cancer 2011;2011:502723.

2 Staples MP, Elwood M, Burton RC, et al. Nonmelanoma skin cancer in Australia: the 2002 national survey and trends since 1985. Med J Aust 2006;184: $6-10$.

3 Chu MB, Slutsky JB, Dhandha MM, et al. Evaluation of the definitions of 'high-risk' cutaneous squamous cell carcinoma using the American Joint Committee on Cancer Staging Criteria and National Comprehensive Cancer Network Guidelines. J Skin Cancer 2014;2014:8.

4 Brantsch KD, Meisner C, Schönfisch B, et al. Analysis of risk factors determining prognosis of cutaneous squamous-cell carcinoma: a prospective study. Lancet Oncol 2008;9:713-720.

5 Brunner M, Ng BC, Veness MJ, et al. Assessment of the new nodal classification for cutaneous squamous cell carcinoma and its effect on patient stratification. Head Neck 2015;37:336-339.

6 Lo JA, Fisher DE. The melanoma revolution: from UV carcinogenesis to a new era in therapeutics. Science 2014;346:945-949.

7 Travis WD, Brambilla E, Riely GJ. New pathologic classification of lung cancer: relevance for clinical practice and clinical trials. J Clin Oncol 2013;31: 992-1001.

8 Edge SB, Compton CC. The American Joint Committee on Cancer: the 7th Edition of the AJCC Cancer Staging Manual and the Future of TNM. Ann Surg Oncol 2010;17:1471-1474.

9 Adzhubei IA, Schmidt S, Peshkin L, et al. A method and server for predicting damaging missense mutations. Nat Methods 2010;7:248-249.

10 Kumar P, Henikoff S, Ng PC. Predicting the effects of coding non-synonymous variants on protein function using the SIFT algorithm. Nat Protoc 2009;4: 1073-1081.

11 Forbes SA, Beare D, Gunasekaran P, et al. COSMIC: exploring the world's knowledge of somatic mutations in human cancer. Nucleic Acids Res 2015;43: D805-D811.

12 Shihab HA, Gough J, Cooper DN, et al. Predicting the functional, molecular, and phenotypic consequences of amino acid substitutions using hidden Markov models. Hum Mutat 2013;34:57-65.

13 Daya-Grosjean L, Sarasin A. The role of UV induced lesions in skin carcinogenesis: an overview of oncogene and tumor suppressor gene modifications in xeroderma pigmentosum skin tumors. Mutat Res 2005;571:43-56.

14 Pickering CR, Zhou JH, Lee JJ, et al. Mutational landscape of aggressive cutaneous squamous cell carcinoma. Clin Cancer Res 2014;20:6582-6592.

15 Kim M-Y, Park HJ, Baek S-C, et al. Mutations of the p53 and PTCH gene in basal cell carcinomas: UV mutation signature and strand bias. J Dermatol Sci 2002;29:1-9.

16 Miyoshi Y, Nagase H, Ando H, et al. Somatic mutations of the APC gene in colorectal tumors: mutation cluster region in the APC gene. Hum Mol Genet 1992;1: 229-233.

$17 \mathrm{Yu}$ J, Wu WKK, Li X, et al. Novel recurrently mutated genes and a prognostic mutation signature in colorectal cancer. Gut 2015;64:636-645.

18 Fang NY, Greiner TC, Weisenburger DD, et al. Oligonucleotide microarrays demonstrate the highest frequency of ATM mutations in the mantle cell subtype of lymphoma. Proc Natl Acad Sci USA 2003;100: 5372-5377.

$19 \mathrm{Wu}$ X, Platt JL, Cascalho M. Dimerization of MLH1 and PMS2 limits nuclear localization of MutL $\alpha$. Mol Cell Biol 2003;23:3320-3328.

20 Bornstein S, White R, Malkoski S, et al. Smad4 loss in mice causes spontaneous head and neck cancer with increased genomic instability and inflammation. J Clin Invest 2009;119:3408-3419.

21 Sos ML, Koker M, Weir BA, et al. PTEN loss contributes to erlotinib resistance in EGFR-mutant lung cancer by activation of Akt and EGFR. Can Res 2009;69: 3256-3261.

22 Snietura M, Jaworska M, Mlynarczyk-Liszka J, et al. PTEN as a prognostic and predictive marker in postoperative radiotherapy for squamous cell cancer of the head and neck. PLoS ONE 2012;7:e33396.

23 Durinck S, Ho C, Wang NJ, et al. Temporal dissection of tumorigenesis in primary cancers. Cancer Discov 2011;1:137-143.

24 Li YY, Hanna GJ, Laga AC, et al. Genomic analysis of metastatic cutaneous squamous cell carcinoma. Clin Cancer Res 2015;21:1447-1456.

25 Silva IP, Salhi A, Giles KM, et al. Identification of a novel pathogenic germline KDR variant in melanoma. Clin Cancer Res 2016;22:2377-2385.

26 Garrido M, Bastian BC. KIT as a therapeutic target in melanoma. J Invest Dermatol 2010;130:20-27.

27 Massarelli E, Johnson FM, Erickson HS, et al. Uncommon epidermal growth factor receptor mutations in non-small cell lung cancer and their mechanisms of EGFR tyrosine kinase inhibitors sensitivity and resistance. Lung Cancer 2013;80:235-241.

28 Tartaglia M, Niemeyer CM, Fragale A, et al. Somatic mutations in PTPN11 in juvenile myelomonocytic leukemia, myelodysplastic syndromes and acute myeloid leukemia. Nat Genet 2003;34:148-150.

29 Chen H, Ma J, Li W, et al. A 'molecular brake' in the kinase hinge region regulates the activity of receptor tyrosine kinases. Mol Cell 2007;27:717-730.

30 Byron SA, Gartside M, Powell MA, et al. FGFR2 point mutations in 466 endometrioid endometrial tumors: relationship with MSI, KRAS, PIK3CA, CTNNB1 
mutations and clinicopathological features. PLoS ONE 2012;7:e30801.

31 Mockus SM, Potter CS, Stafford GA, et al. Abstract 73: targeting KDR mutations in lung adenocarcinoma. Cancer Res 2015;75:73.

32 Leiser D, Medová M, Mikami K, et al. KRAS and HRAS mutations confer resistance to MET targeting in preclinical models of MET-expressing tumor cells. Mol Oncol 2015;9:1434-1446.

33 Shima F, Yoshikawa Y, Ye M, et al. In silico discovery of small-molecule Ras inhibitors that display antitumor activity by blocking the Ras-effector interaction. Proc Natl Acad Sci USA 2013;110:8182-8187.

34 Atwood SX, Sarin KY, Whitson RJ, et al. Smoothened variants explain the majority of drug resistance in basal cell carcinoma. Cancer Cell 2015;27:342-353.

35 Martincorena I, Roshan A, Gerstung M, et al. High burden and pervasive positive selection of somatic mutations in normal human skin. Science (New York, NY) 2015;348:880-886.

36 Jonason AS, Kunala S, Price GJ, et al. Frequent clones of p53-mutated keratinocytes in normal human skin. Proc Natl Acad Sci USA 1996;93:14025-14029.

37 Lapke N, Lu Y-J, Liao C-T, et al. Missense mutations in the TP53 DNA-binding domain predict outcomes in patients with advanced oral cavity squamous cell carcinoma. Oncotarget 2016;7:44194-44210.

38 Maréchal A, Zou L. DNA damage sensing by the ATM and ATR kinases. Cold Spring Harb Perspect Biol 2013;5:a012716.

39 Ismail F, Ikram M, Purdie K, et al. Cutaneous squamous cell carcinoma (SCC) and the DNA damage response: pATM expression patterns in pre-malignant and malignant keratinocyte skin lesions. PLoS ONE 2011;6:e21271.

40 Ponti G, de Leon MP. Muir-Torre syndrome. Lancet Oncol 2005;6:980-987.

41 Yoon G, Lee H, Kim J-H, et al. Clinical significance of fibroblast growth factor receptor 2 expression in patients with residual rectal cancer after preoperative chemoradiotherapy: relationship with KRAS or BRAF mutations and MSI status. Tumor Biol 2016;37: 10209-10218.

42 Li C-F, He H-L, Wang J-Y, et al. Fibroblast growth factor receptor 2 overexpression is predictive of poor prognosis in rectal cancer patients receiving neoadjuvant chemoradiotherapy. J Clin Pathol 2014;67:1056-1061.

$43 \mathrm{Su}$ X, Zhan P, Gavine PR, et al. FGFR2 amplification has prognostic significance in gastric cancer: results from a large international multicentre study. $\mathrm{Br} \mathrm{J}$ Cancer 2014;110:967-975.

44 South AP, Purdie KJ, Watt SA, et al. NOTCH1 mutations occur early during cutaneous squamous cell carcinogenesis. J Invest Dermatol 2014;134:2630-2638.

45 Reichrath J, Reichrath SNotch-signaling and nonmelanoma skin cancer: an ancient friend, revisitedIn:Reichrath J, Reichrath Seditors. Notch Signaling in Embryology and Cancer. Springer: New York, NY, USA, 2012, pp 265-271.

46 Groth C, Fortini ME. Therapeutic approaches to modulating Notch signaling: current challenges and future prospects. Semin Cell Dev Biol 2012;23: 465-472.

47 Al-Rohil RN, Tarasen AJ, Carlson JA, et al. Evaluation of 122 advanced-stage cutaneous squamous cell carcinomas by comprehensive genomic profiling opens the door for new routes to targeted therapies. Cancer 2016;122:249-257.

48 Lewis CM, Glisson BS, Feng L, et al. A phase II study of gefitinib for aggressive cutaneous squamous cell carcinoma of the head and neck. Clin Cancer Res 2012;18: 1435-1446.

49 Maubec E, Petrow P, Scheer-Senyarich I, et al. Phase II study of cetuximab as first-line single-drug therapy in patients with unresectable squamous cell carcinoma of the skin. J Clin Oncol 2011;29:3419-3426.

50 Euvrard S, Morelon E, Rostaing L, et al. Sirolimus and secondary skin-cancer prevention in kidney transplantation. N Engl J Med 2012;367:329-339.

Supplementary Information accompanies the paper on Modern Pathology website (http://www.nature.com/ modpathol) 